\title{
BMJ Global Health The secondary transmission pattern of COVID-19 based on contact tracing in Rwanda
}

Muhammed Semakula, ${ }^{1,2}$ FranÇois Niragire, ${ }^{3}$ Angela Umutoni, ${ }^{4}$ Sabin Nsanzimana, ${ }^{4}$ Vedaste Ndahindwa, ${ }^{5}$ Edison Rwagasore, ${ }^{6}$ Thierry Nyatanyi, ${ }^{4}$ Eric Remera (D) , ${ }^{4}$ Christel Faes ${ }^{7}$

To cite: Semakula $M$, Niragire FÇ, Umutoni A, et al. The secondary transmission pattern of COVID-19 based on contact tracing in Rwanda. BMJ Global Health 2021;6:e004885. doi:10.1136/ bmjgh-2020-004885

Handling editor Seye Abimbola

- Additional supplemental material is published online only. To view, please visit the journal online (http://dx.doi.org/10. 1136/bmjgh-2020-004885)

Received 27 January 2021 Revised 1 April 2021 Accepted 5 April 2021

\section{Check for updates}

(c) Author(s) (or their employer(s)) 2021. Re-use permitted under CC BY-NC. No commercial re-use. See rights and permissions. Published by BMJ.

For numbered affiliations see end of article.

Correspondence to Muhammed Semakula; semakulam@gmail.com

\section{ABSTRACT}

Introduction COVID-19 has shown an exceptionally high spread rate across and within countries worldwide. Understanding the dynamics of such an infectious disease transmission is critical for devising strategies to control its spread. In particular, Rwanda was one of the African countries that started COVID-19 preparedness early in January 2020, and a total lockdown was imposed when the country had only 18 COVID-19 confirmed cases known. Using intensive contact tracing, several infections were identified, with the majority of them being returning travellers and their close contacts. We used the contact tracing data in Rwanda for understanding the geographic patterns of COVID-19 to inform targeted interventions.

Methods We estimated the attack rates and identified risk factors associated to COVID-19 spread. We used Bayesian disease mapping models to assess the spatial pattern of COVID-19 and to identify areas characterised by unusually high or low relative risk. In addition, we used multiple variable conditional logistic regression to assess the impact of the risk factors.

Results The results showed that COVID-19 cases in Rwanda are localised mainly in the central regions and in the southwest of Rwanda and that some clusters occurred in the northeast of Rwanda. Relationship to the index case, being male and coworkers are the important risk factors for COVID-19 transmission in Rwanda.

Conclusion The analysis of contact tracing data using spatial modelling allowed us to identify high-risk areas at subnational level in Rwanda. Estimating risk factors for infection with SARS-CoV-2 is vital in identifying the clusters in low spread of SARS-CoV-2 subnational level. It is imperative to understand the interactions between the index case and contacts to identify superspreaders, risk factors and high-risk places. The findings recommend that self-isolation at home in Rwanda should be reviewed to limit secondary cases from the same households and spatiotemporal analysis should be introduced in routine monitoring of COVID-19 in Rwanda for policy making decision on real time.

\section{INTRODUCTION}

Rwanda was one of the first African country to prepare against the spread of COVID-19

\section{Key questions}

What is already known?

- The contact tracing is important to curb the transmission of SARS-CoV-2- Understanding the spatial distribution of COVID-19 is crucial to control new infections

What are the new findings?

- We identified the secondary transmission pattern of COVID-19 using spatial models

- We estimated the Risk factors for infections with SARS-CoV-2

What do the new findings imply?

- The findings suggested to review self-isolation at home policy in Rwanda to limit secondary transmission from the same household.

- The findings suggested to include spatial and spatiotemporal analysis in routine monitoring of COVID-19 for policy making decision on real time.

in January 2020 by screening and recording everyone who travelled to China or in any other country that had a declared COVID-19 case. A total lockdown was imposed when the country had only 18 COVID-19 confirmed cases on 22 March 2020. Using intensive contact tracing, several COVID-19 cases were identified, with the majority of them among returning travellers and contacts of identified cases. Lockdown and contact tracing strategies interrupted the chain of transmission and provided time to the Rwanda COVID-19 Joint Task Force to establish treatment centres and allow readiness of response teams. The contact tracing has a long history in Rwanda and has played a major role in controlling various infectious diseases including Ebola virus disease, severe acute respiratory syndrome and Middle East upper respiratory syndrome. ${ }^{1}$ Several of these more recent outbreaks have occurred on the 
African continent, allowing African scientists and public health officials to build their teams and capacity. ${ }^{2}$

As part of Rwanda's COVID-19 response, the government applied a comprehensive surveillance approach that includes screening, testing and isolation (quarantine) on real time using innovative technology. It defines a suspect case, a confirmed case and a contact as follows. A suspect COVID-19 case is a patient with acute respiratory illness such as fever and at least one symptom of respiratory disease (cough, shortness of breath, fatigue, anorexia, myalgia, headache, rhinorrhoea, sore throat, vomiting, diarrhoea, smell and taste disorders, clinical signs of pneumonia and abnormal chest) or someone who has travelled in the last 14 days to an area that has reported confirmed case(s) of COVID-19. ${ }^{3}$ A confirmed COVID-19 case is a person with laboratory confirmation of COVID-19 infection using reverse transcription PCR testing for SARS-CoV-2, irrespective of clinical signs and symptoms. A contact of a COVID-19 case was defined as any person who had contact with a COVID-19 case within a timeframe ranging from 72 hours before the onset of symptoms of the case to 14 days after the onset of symptoms. ${ }^{4}$

Rwanda's contact tracing strategy was to test each suspect COVID-19 case and trace all contacts, the travel history and visited places in the past 14 days for each confirmed case by a protected epidemiologist. Each identified contact was tested at least twice within a period of 2 weeks. In this way, Rwanda has traced more than 10 000 contacts between 14 March 2020 and 20 July 2020, with the aim to identify potentially infected persons and prevent onward transmissions of secondary cases. The first 300 COVID-19 cases identified in Rwanda were predominately imported cases, and most of them were sent to quarantine sites immediately on arrival at airport. Despite early detection strategies in place, some imported cases were identified after being in contact with their families, relatives, friends and coworkers.

For a better management and control of COVID-19, understanding the geographical risk regions is the primary and fundamental step to take. ${ }^{5}$ Spatial analysis is of great help in understanding the spread of infectious diseases, spatial association and predicting local outbreaks. Spatial analysis is critical to develop public health policies during early stages of COVID-19. ${ }^{6}$ Until now, studies evaluating the spatial spread of the COVID-19 pandemic in Rwanda are limited. However, this study contributes on identifying high-risk areas in Rwanda at subnational level.

Furthermore, estimating the secondary attack rate (SAR) is important in epidemiology. Attack rates may contribute significantly in designing and developing infection control and prevention policies for COVID-19 that can contribute to limit the spread of the disease. The SAR is defined as the ratio of the number of cases occurring within the incubation period on exposure to a primary case and the total susceptible contacts. ${ }^{7}$

In this study, we used contact tracing data in Rwanda to understand geographic patterns of COVID-19 to inform targeted interventions, estimate SARs and identify factors associated with increased transmission probability among contacts of COVID-19 index cases. Findings from our study will help identify high-risk areas and groups and provide evidence to develop targeted interventions to reduce the burden of COVID-19 in Rwanda and similar settings.

\section{METHODS \\ Data source}

We have used COVID-19 contact tracing data, recorded from 14 March 2020 to 20 July 2020, from the Rwanda health management information system (RHMIS). The data set contains 2216 index cases, among them 626 cases were identified and isolated in government-supervised isolation centre before meeting anyone at point of entries. Only 1590 have completed all details, and their contacts (11 809) were identified through contact tracing.

Rwanda has a digitised real-time COVID-19 data system. Everyone tested COVID-19 positive, laboratory system automatically sends his or her details to contact tracing system that enables the tracers to start investigation immediately. ${ }^{8}$ Investigation is performed by interviewing persons at the treatment centre or at home for those under home-based care programme. However, extra information about contacts are also generated through algorithms in place that are using combination of telephone numbers and network connectivity used by a positive case in the last 7 days prior to test positive. Once all contacts are identified, the tracers record their details in the system and are followed up for a period of 7-14 days from the last day they met a confirmed COVID-19 case. ${ }^{4}$ All contacts are tested at least twice during the follow-up period. For both cases and contacts, detailed demographics, clinical diagnostics, laboratory details, travel history, employment details, residence and people living with him/her are recorded in the contact tracing data system hosted at Rwanda National Data Center under management of Rwanda Biomedical Centre.

Figure 1 shows an example of the systematic contact tracing of one confirmed case that generated seven secondary cases: two secondary cases and five tertiary cases. The black cycles in figure 1 are positive cases and include the case numbers and the date that the cases tested positive. Red, yellow and green cycles are the contacts identified, investigated and tested, and correspond to high-risk, medium-risk and low-risk contacts. A similar exercise was done to each and every positive case in Rwanda. Rwanda focused much on contact tracing, but unfortunately, less attention has been paid to the risk factors related to COVID-19 transmission.

\section{Statistical analysis}

The attack rate was estimated as the percentage of contacts who were later confirmed to be infected with SARS-CoV-2 among all secondary contacts of the index case. The estimation was presented by gender, age, the 


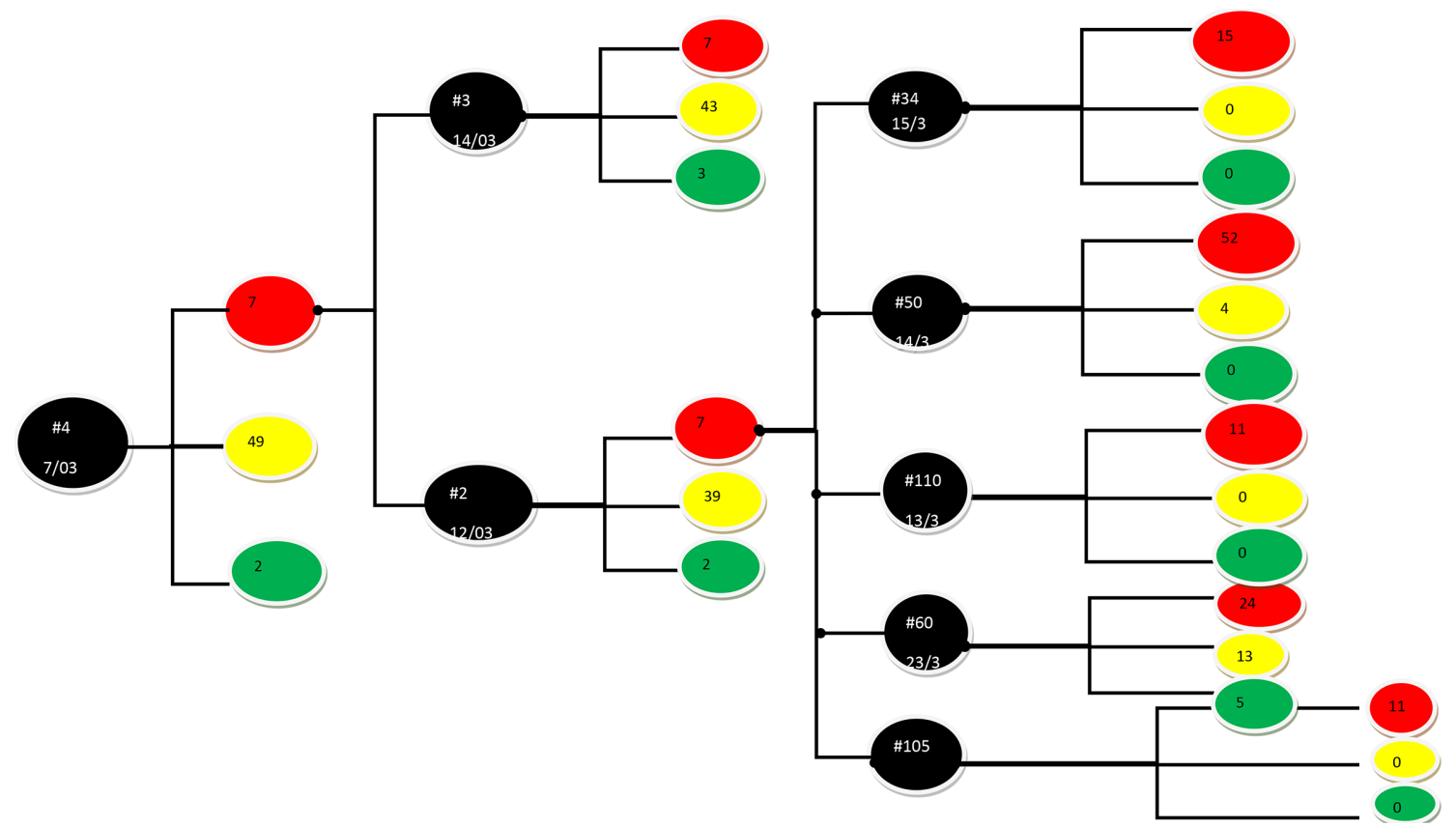

Figure 1 COVID-19 systematic contacts tracing of case number 4 .

type of relationship to the index case, place of infection and symptoms. Moreover, we explored the age distribution of the index cases and their contacts, as well as of the secondary cases to see which age groups are most likely to interact.

Standard non-parametric tests were used, mainly Fisher's exact test, the Kruskal-Wallis and the Mann-Whitney $\mathrm{U}$ test to compare characteristics between demographic groups. ${ }^{9}$ We used Bayesian disease mapping models to assess the spatial pattern of COVID-19 and to identify areas characterised by unusually high or low relative risk. In addition, we used multiple variable conditional logistic regression models without interaction term (M1) and with interaction term (M2) to identify factors associated with COVID-19 infection. We calculated the standardised incidence ratio (SIR) in each area taking the age and sex structure in an area into consideration to investigate the COVID-19 risk in an area. ${ }^{10-12}$ We extended our methods to take into account spatial dependence among neighbouring areas using Bayesian disease mapping approaches.

A Bayesian disease mapping model consists of three components: the data model (ie, the distribution of the data given the parameters), the process model (ie, a description of underlying spatial trend) and the parameter model (ie, the prior distribution of the parameters to be estimated) ${ }^{13}$ The data model is given by

$$
\text { Yit } \sim \text { Poisson (Eit } \theta \text { it); }
$$

where a Poisson distribution is appropriate since disease data are counts of number of cases and are non-negative. It is assumed that the mean is a product of the expected count Eit and the relative risk $\theta$ it. The process model describes the underlying structure of the relative risks. We used the Besag-York-Mollie model, which is the CAR convolution model with two random effects, one spatially structured area-specific random effect and one unstructured area-specific random effect ${ }^{14} 15$

$$
\log (\theta \mathbf{i})=\alpha+\mathbf{u i}+\nu \mathbf{i}
$$

where, ui is the spatially structured area-specific random effect that allows for smoothing among adjacent areas, namely $^{14}$

$$
u_{i} \mid u_{j} \sim N\left(\bar{\mu} \delta_{i}, \frac{\sigma_{u}^{2}}{n \delta_{i}}\right)
$$

With $\delta \_i$ and $n \_\delta i$, respectively, the set of neighbours and number of neighbours for a specific area $i$. The unstructured component $\mathrm{v} \_\mathrm{i}$ is modelled using as a Gaussian process

$$
u_{i} \sim N\left(0, \sigma_{u}^{2}\right)
$$

and allows for extra heterogeneity in the counts due to unobserved (and spatially unstructured) risk factors. We have used Integrated Nested Laplace approximation for estimation of this model. ${ }^{16}$ We make use of excess probability to investigate the hotspots. ${ }^{10}{ }^{17}$ Furthermore, to identify individual risk factors, logistic regression is used with multiple explanatory variables, which took into consideration the interaction terms of the age and the type of relationship to the index case. ${ }^{18}$ The response variable is whether the secondary case tested positive or negative to COVID-19, which is a binary outcome for each contact to the index case and which we denote as $\mathrm{Z}$. The probability of testing COVID-19 positive is defined as $\varpi$, and consequently, the probability of testing negative is $1-\varpi$. The multiple logistic regression equation can be written as Z binomial(pi) with

$$
\operatorname{logit}(\pi)=\ln \left(\frac{\pi}{1-\pi}\right)=\beta_{0}+\beta_{1} x_{1}+\beta_{2} x_{2}+\ldots+\beta_{p} x_{p}(3)
$$

with $\mathrm{p}$ the number of independent variables (gender, age, relationship to the index cases and intersections between the independent variables). $\beta_{0}$ is the intercept 
and quantifies the probability of infection in the reference group, that is, the group that takes on the value 0 for all variables; $\beta_{i}$ is the regression coefficient corresponding to risk factor $x_{i}$, with $\exp$ (beta_i) the multiplicative increase or decrease in the odds to test positive for a one unit increase in $x_{i}$, while all other covariates remain unchanged.

The probability of testing positive can be written as

$$
\pi=\frac{\exp \left(\beta_{0}+\beta_{1 \times 1}+\beta_{2 x 2}+\beta_{3 x 3}+\ldots+\beta_{i x i}\right)}{1+\exp \left(\beta_{0}+\beta_{1 \times 1}+\beta_{2 x 2}+\beta_{3 x 3}+\ldots+\beta_{i x i}\right)}
$$

The regression parameters are estimated using maximum likelihood. The regression coefficient $\beta_{i}$ are transformed into OR by taking $\exp \left(\beta_{i}\right)$. The OR value reflects the strength of association between exposure and the outcome. $^{18}$

For the goodness of fit of multiple variable logistic models, we used likelihood ratio test. The likelihood ratio statistic $\mathrm{G}^{2}(\mathrm{M} 0 \mid \mathrm{M} 1)=-2\left(\mathrm{~L}_{0}-\mathrm{L}_{1}\right)$ was defined to test whether model parameters are zero, given that M1 holds, by comparing the log likelihood L1 for fitted model M1 with

L0 for a simpler model M0.

\section{Patient and public involvement}

Data used for this study were anonymised, deidentified and routinely collected during outbreak investigation and response data maintained by RHMIS, under Public Health Surveillance and Epidemic Preparedness and Response Division of Rwanda Biomedical Center. No participants were involved directly in data collection; therefore, their consent was waived by Rwanda National Ethics Committee, which also approved the use of outbreak and investigation team presented here. The Rwanda Ministry of Health also granted approval for data access and use to the principal investigator
(MS) for the purpose of improving COVID-19 contact tracing in Rwanda.

\section{RESULTS}

The results are presented in two parts. The first part includes COVID-19 attack rate by exposure routes. The second part provides a geographical analysis of the number of contacts and secondary cases.

\section{COVID-19 attack rate and related risk factors}

The mean number of contacts per index cases was 7, though the number of contacts per case ranges from 0 to 301 contacts (IQR 0-9, figure 2A). We have observed five index cases with more than 200 contacts in less than 1 week. The mean number of secondary cases per index case was 0.25 (IQR $0-0$ and range $0-6$, figure $2 \mathrm{~B}$ ). The individual SAR, defined as the ratio of the number of secondary cases to the number of contact, ranges from 0.0 to 0.5 , with mean equal to 0.017 (IQR 0.0-0.0, figure 2C). The index case that had 301 contacts was among one of the superspreaders of COVID-19, with five of his contacts tested positive. Another superspreader in this study was a school teacher with 96 contacts, resulting in six positive secondary cases.

We have provided the demographic details of the index cases and their contacts in table 1. With 1590 index cases, we traced 11809 contacts of which 209 are secondary cases and 11600 uninfected contacts. The overall attack rate is $1.77 \%$ (95\% CI $1.55 \%$ to $2.02 \%$ ). The majority of both the index cases $(76.13 \%)$ and the contacts $(51.35 \%)$ were male and aged between 20 and 39 years. The SAR for males is $2.06 \%$ (95\% CI $1.73 \%$ to $2.46 \%$ ), slightly higher as compared with females with overall
A

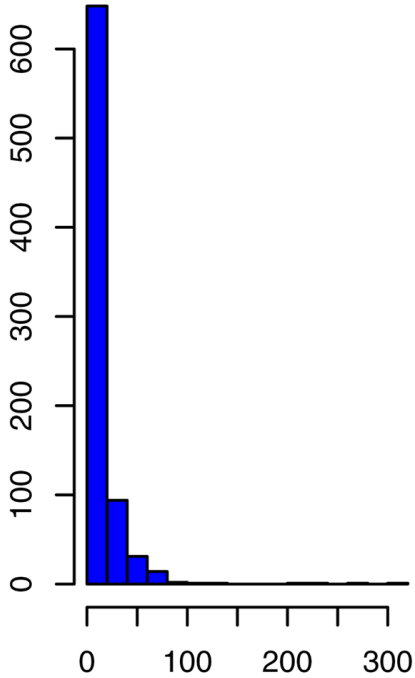

Number of Contacts per index Cases

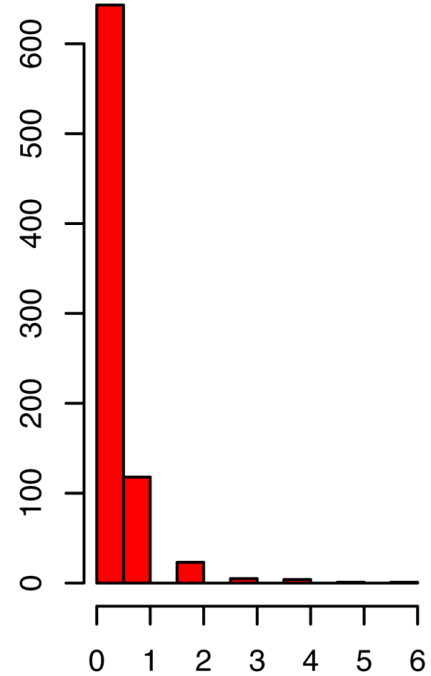

Secondary cases per index cases

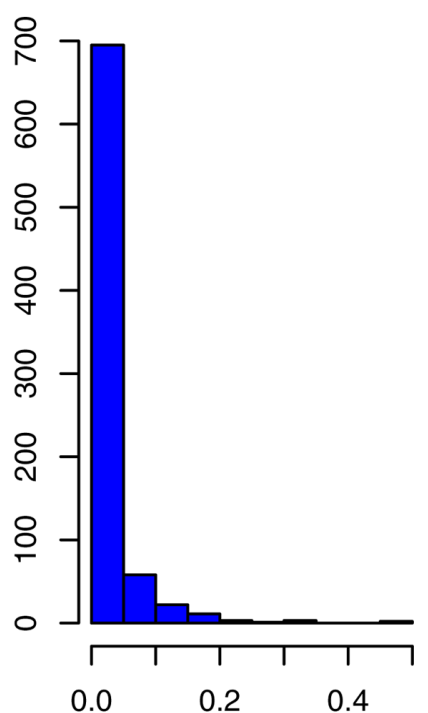

AR

Figure 2 COVID-19 contacts tracing: the number of contacts per index case (left), the number of secondary cases per index case (middle) and the individual COVID-19 attack rates (right). 
Table 1 Demographic characteristic of the study population and data-based secondary

\begin{tabular}{|c|c|c|c|c|}
\hline $\begin{array}{l}\text { Demographic } \\
\text { characteristics }\end{array}$ & $\begin{array}{l}\text { Index case } \\
\text { n (\%) }\end{array}$ & $\begin{array}{l}\text { Contacts of } \\
\text { index cases } \\
\mathrm{n}(\%)\end{array}$ & $\begin{array}{l}\text { Contacts } \\
\text { positive } \\
\text { n (\%) }\end{array}$ & $\begin{array}{l}\text { Secondary } \\
\text { attack rate } \\
\%(95 \% \mathrm{Cl})\end{array}$ \\
\hline \multicolumn{5}{|l|}{ Gender } \\
\hline Female & $529(23.87)$ & 5745 (48.65) & $84(40.19)$ & 1.46 (1.17 to 1.82$)$ \\
\hline Male & $1687(76.13)$ & $6064(51.35)$ & $125(59.81)$ & 2.06 (1.73 to 2.46$)$ \\
\hline \multicolumn{5}{|l|}{ Age group (years) } \\
\hline $0-9$ & $36(1.6)$ & $1553(13.15)$ & $25(11.96)$ & 1.61 (1.07 to 2.40$)$ \\
\hline $10-19$ & $230(10.38)$ & $1511(12.80)$ & $26(12.44)$ & 1.72 (1.14 to 2.55$)$ \\
\hline $20-29$ & $665(30.01)$ & 2471 (20.92) & $62(29.67)$ & 2.51 (1.94 to 3.23 ) \\
\hline $30-39$ & $651(29.38)$ & $2444(20.70)$ & $51(24.40)$ & 2.09 (1.57 to 2.76$)$ \\
\hline $40-49$ & $309(13.98)$ & $1741(14.74)$ & $29(13.88)$ & 1.67 (1.14 to 2.42$)$ \\
\hline $50-59$ & $172(7.76)$ & $1341(11.36)$ & $15(7.18)$ & 1.12 (0.65 to 1.88$)$ \\
\hline$>60$ & $153(6.9)$ & $748(6.33)$ & $1(0.48)$ & 0.13 (0.01 to 0.86$)$ \\
\hline \multicolumn{5}{|c|}{ Evacuated to isolation centre } \\
\hline No & $1946(87.82)$ & 11211 (94.94) & 199 (94.94) & 1.78 (1.55 to 2.04$)$ \\
\hline Yes & $270(12.18)$ & $598(5.06)$ & $10(5.06)$ & 1.67 (0.90 to 3.08$)$ \\
\hline \multicolumn{5}{|c|}{ Travelled in other countries } \\
\hline Travelled & $246(11.10)$ & & & \\
\hline Not travelled & $1970(88.90)$ & & & \\
\hline \multicolumn{5}{|l|}{ Relationship to index } \\
\hline Household & & $615(5.21)$ & $18(8.61)$ & 2.93 (1.84 to 4.60$)$ \\
\hline Market/shop/pub & & $9191(77.83)$ & $146(69.86)$ & 1.59 (1.35 to 1.86$)$ \\
\hline Transport & & $142(1.20)$ & $3(1.44)$ & 2.11 (0.67 to 6.43 ) \\
\hline Social & & $1273(10.78)$ & 27 (12.92) & 2.12 (1.46 to 0.31$)$ \\
\hline Coworkers & & $588(4.98)$ & $15(7.18)$ & 2.55 (1.54 to 4.19$)$ \\
\hline \multicolumn{5}{|l|}{ Had symptoms } \\
\hline No symptoms & & 11531 (97.65) & 203 (97.13) & 1.74 (1.52 to 2.01$)$ \\
\hline With symptoms & & $278(2.35)$ & $6(2.87)$ & 3.73 (1.54 to 8.74$)$ \\
\hline \multicolumn{5}{|l|}{ Type of symptoms } \\
\hline Fever & & $9(0.08)$ & $1(0.48)$ & 11.11 (0.92 to 62.60$)$ \\
\hline Difficulty breathing & & $11(0.09)$ & 0 & 0 \\
\hline Runny nose & & $18(0.15)$ & 0 & 0 \\
\hline Throat & & $10(0.08)$ & 0 & 0 \\
\hline Cough & & $237(2.01)$ & $1(0.48)$ & 0.42 (0.059 to 2.97$)$ \\
\hline Chest pain & & $40(0.34)$ & $5(2.39)$ & 12.5 (5.09 to 27.55$)$ \\
\hline None & & $11484(97.25)$ & 202 (96.65) & 1.76 (1.53 to 2.02$)$ \\
\hline Total & $2216(100)$ & $11809(100)$ & $209(100)$ & 1.77 (1.55 to 2.02$)$ \\
\hline
\end{tabular}

attack rate of $1.46 \%$ (95\% CI $1.17 \%$ to $1.82 \%$ ). The SAR for the age group 20-39 years is highest among all age groups, with attack rate 2.51 (95\% CI 1.94 to 3.23). Only $270(12.18 \%)$ if the primary cases and $598(5.06 \%)$ of the secondary cases were isolated at government supervised isolation centres, while all others were self-isolated at their home for a period of 7-14 days as per Rwanda COVID-19 protocol.
About $5.21 \%$ of the contacts were household contacts, with an overall SAR of $2.93 \%$ (95\% CI $1.85 \%$ to $4.60 \%$ ). The most common contacts $(77.83 \%)$ were people that met in public spaces such as open markets, shops or pubs. The SAR for these contacts is 1.59 (95\% CI 1.35 to 1.86). The second most common type of contact are contacts in social activities $(10.78 \%)$, with a SAR of $2.12 \%$ (95\% CI $1.46 \%$ to $3.08 \%$ ). Less common are contact in vehicles $(1.20 \%)$, with an attack 
Table 2 Logistic regression model SARS-CoV-2: risk factors associated to secondary infection

\begin{tabular}{|c|c|c|c|c|}
\hline \multirow[b]{2}{*}{ Covariates } & \multicolumn{2}{|c|}{ Model without interaction } & \multicolumn{2}{|c|}{ Model with both effect } \\
\hline & $\begin{array}{l}\text { M1 } \\
\text { OR (95\% Cl) }\end{array}$ & $P$ value & $\begin{array}{l}\text { M2 } \\
\text { OR (95\% Cl) }\end{array}$ & $P$ value \\
\hline \multicolumn{5}{|l|}{ Gender of index case (ref. females) } \\
\hline Male & 1.43 (1.08 to 1.89$)$ & 0.013 & 1.43 (1.08 to1.89) & 0.012 \\
\hline \multicolumn{5}{|c|}{$\begin{array}{l}\text { Age category of index case (ref. <20) } \\
\text { (years) }\end{array}$} \\
\hline $20-49$ & 1.27 (0.92 to1.76) & 0.146 & $1.22(0.83$ to 1.79$)$ & 0.311 \\
\hline $50+$ & 0.45 (0.26 to 0.79$)$ & 0.006 & 0.49 (0.25 to 0.94$)$ & 0.032 \\
\hline \multicolumn{5}{|l|}{ Relationship (ref. market/shop/pub) } \\
\hline HH members & $1.92(1.17$ to 3.15$)$ & 0.01 & $0.87(0.21$ to 3.64$)$ & 0.846 \\
\hline Transport & $1.44(0.45$ to 4.58$)$ & 0.538 & $1.22(0.16$ to 9.09$)$ & 0.844 \\
\hline Social activity & $1.38(0.91$ to 2.1$)$ & 0.125 & 1.21 (0.5 to 2.88$)$ & 0.674 \\
\hline Workmates & 1.69 (0.98 to 2.89$)$ & 0.057 & $2.8(1.16$ to 6.76$)$ & 0.022 \\
\hline \multicolumn{5}{|c|}{ Relationship and age (ref. market/shop/pub, age <20 years) } \\
\hline Household members \# 20-49 & & & $\begin{array}{l}2.57(0.55 \text { to } \\
12.01)\end{array}$ & 0.231 \\
\hline Household members \# 50+ & & & $\begin{array}{l}2.9(0.36 \text { to } \\
23.24)\end{array}$ & 0.317 \\
\hline Transport \# 20-49 & & & $\begin{array}{l}1.51(0.13 \text { to } \\
17.67)\end{array}$ & 0.743 \\
\hline Transport \# 50+ & & & 1 & \\
\hline Social activity \# 20-49 & & & 1.3 (0.48 to 3.53$)$ & 0.61 \\
\hline Social activity \# 50+ & & & 0.47 (0.05 to 4.38$)$ & 0.51 \\
\hline Coworker \# 20-49 & & & $0.49(0.15$ to 1.53$)$ & 0.217 \\
\hline Coworker \# 50+ & & & 0.39 (0.04 to 3.67$)$ & 0.414 \\
\hline Observations & 11809 & & 11783 & \\
\hline
\end{tabular}

rate of $2.11 \%(95 \%$ CI $0.67 \%$ to $6.42 \%)$, and contact among coworkers $(4.98 \%)$, with an attack rate of $2.55 \%(95 \%$ CI $1.54 \%$ to $4.19 \%)$.

All the contacts were screened for symptoms and 278 of the 11809 contacts $(2.35 \%)$ had symptoms, with the most common symptoms being cough (85.25\%) and chest pain $(14.30 \%)$. However, only few of them tested positive, and among the 209 secondary cases, only 7 showed symptoms.

Some care is needed with the above numbers, as these effects can be confounded by other covariates. Therefore, a multiple logistic regression is performed to investigate the joint effect of gender, age and the type of relationship. From this analysis (table 2), we can see that the OR for male index cases to have a secondary case is higher as compared with a female index case (OR 1.43, 95\% CI 1.08 to 1.89). Contacts of index cases aged 50+ years had lower odds as compared with those below 20 years old in Rwanda (OR 0.45, 95\% CI 0.26 to 0.79 ). The household members of index cases had nearly twice the risk of infection compared with contacts that met index cases in public places such open market, shops or pubs (OR 1.92, 95\% CI 1.17 to 3.15 ). However, the estimates are different for different age groups within the same household, with the OR for index cases aged $<20$ years being lower (OR 0.87, 95\% CI 0.21 to 3.64), and for index cases aged 20, 49 and 50+ years higher (OR 2.57, 95\% 0.55 to 12.01 and OR2.9, $95 \%$ CI 0.36 to 23.24 , respectively). While not statistically significant-possible due to limited number of secondary infections - this hints that there is a difference in contact behaviour depending on the type of contact and age. The overall OR of coworkers as compared with contacts at public places to be infected is 1.69 (95\% CI 0.89 to 2.89 ), though (while not statistically significant) it is higher for index cases $<20$ years of age (OR 2.8, 95\% CI 1.16 to 6.76 ) and smaller for index cases aged 20, 49 or $>50$ years (OR $0.49,95 \%$ CI 0.15 to 1.53 ) and OR $0.39,95 \%$ CI 0.04 to 3.67 , respectively). Also contacts at social activities and on transport are of somewhat higher risk as contacts at public places, though not statistically different.

Finally, we investigate the interaction of age of index case and age of contacts. Therefore, we compared the age groups (0-9, 10-19, 20-29, 30-39, 40-49, 50-59 and $60+$ years) of contacts and index cases. Figure 3 shows the attack rates (ARs) for men and women, with the AR for males being highest among contacts aged 20-29 years and 30-39 years who interact more with index cases aged $50-59$ years or above 60 years. 
AR - males

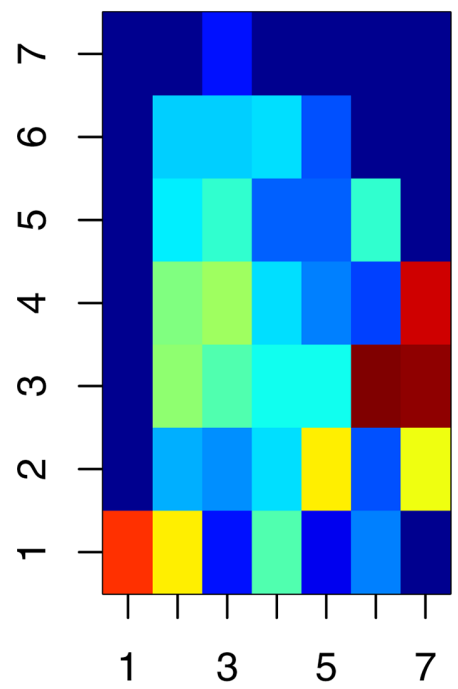

Agegroup index case
AR - females
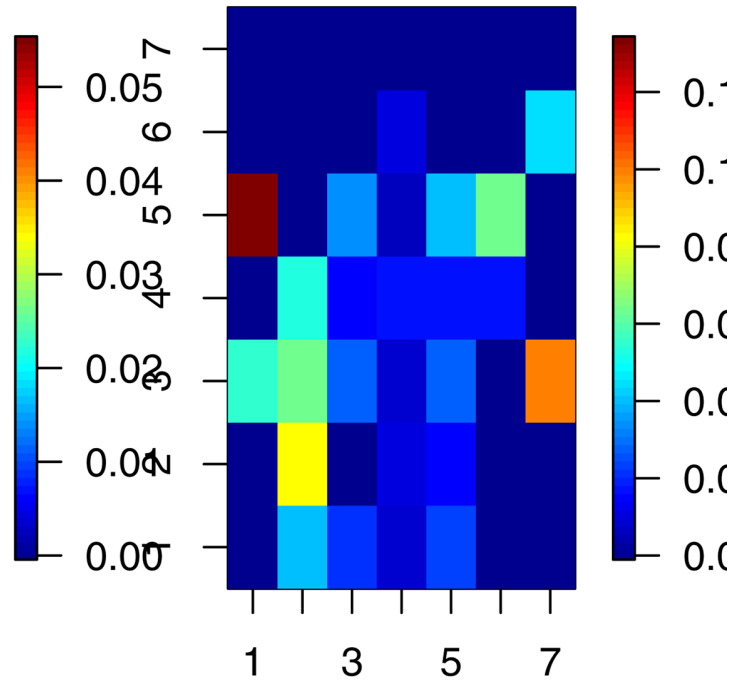

Agegroup index case

Figure 3 Intersection age group and sex: contacts and index cases. AR, attack rate.

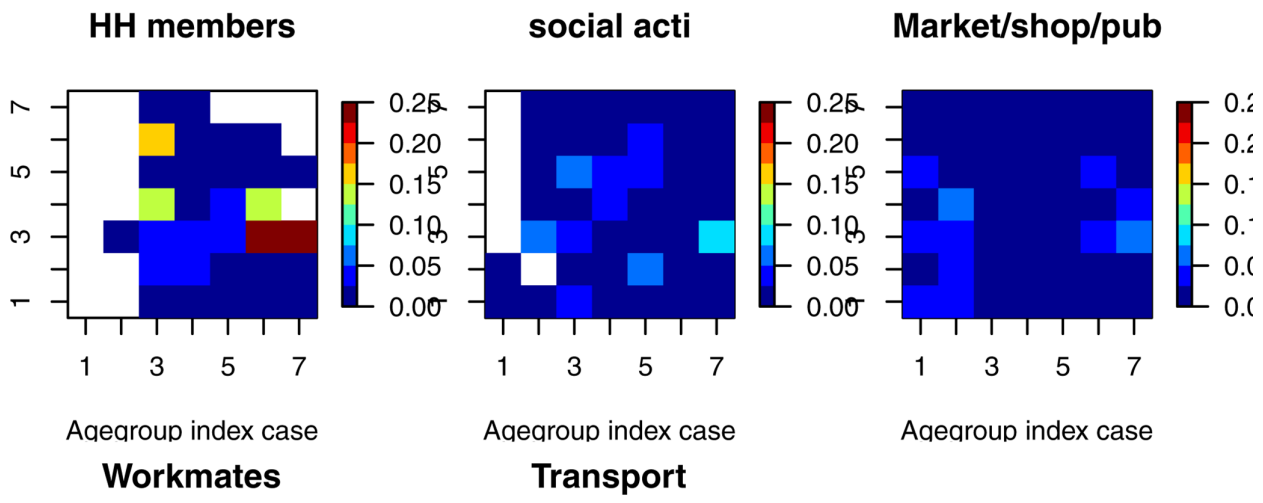

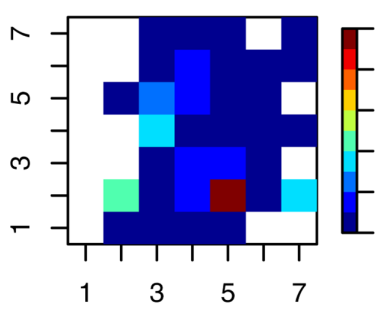

Aqearoup index case

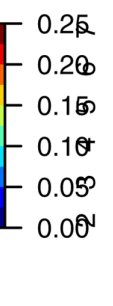

Aatoup index case

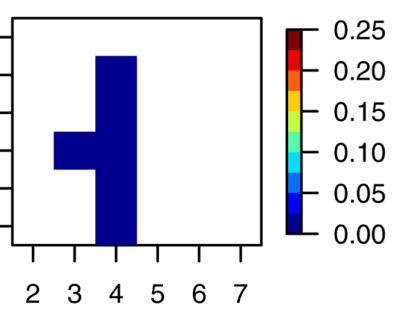

Aqearoup index case

Figure 4 Intersection age group: potential risk factors and attack rate.

Figure 4 shows that the age-age interactions on the risk for secondary infection varies in different contact settings, but was largely influenced by contacts in household settings and among coworkers.

\section{Spatial analysis of contacts and secondary cases}

There are major geographical differences in the risk of making contact to an index case. Figure 5 (top left) shows a geographical presentation of the number of contacts per sector. The three sectors of Rusizi Kamembe, Gihundwe and Mururu recorded the highest number of identified contacts per sector, with the number of contact to an index case being 2509, 1527 and 882, respectively. Gihombo sector in Nyamasheke District (Central-Western) recorded 784 contacts. Most of the sectors in the City of Kigali recorded between 100 and 400 contacts. Details of the top 20 sectors with the highest number of contacts are provided in table 3 , together with the SIR. In some sectors, the risk of making contact to an index case was very high (SIR >10), mainly in the southwestern province. In Rusizi District, more than eight sectors had SIR of above 8. 


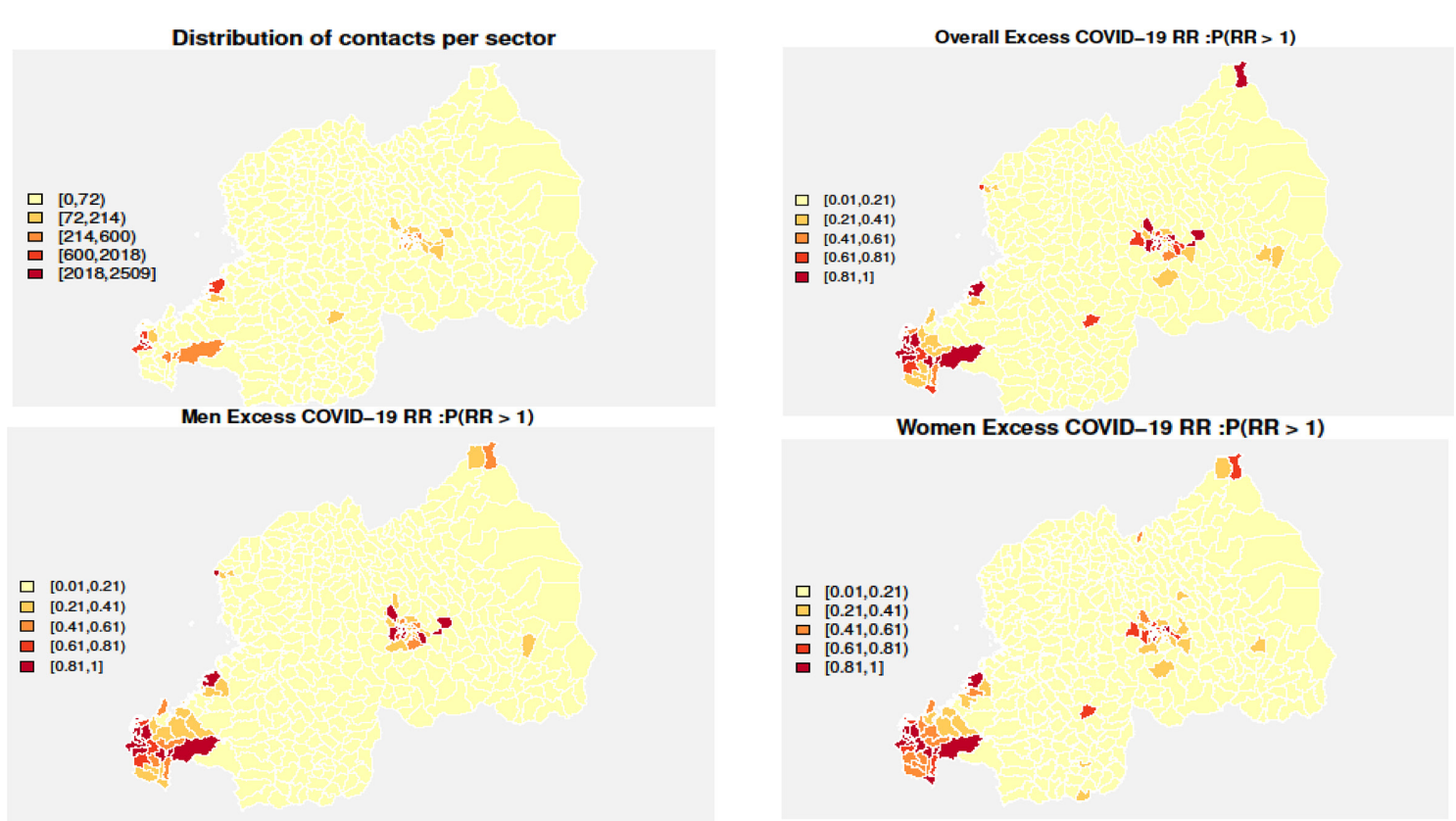

Figure 5 The area-specific probability of COVID-19 case excesses.

The map presented in figure 5 shows that contacts to index cases are clustered mainly in the City of Kigali, which is located in the centre of Rwanda and in the southwest of the country (Rusizi and Nyamasheke Districts). We observe that most COVID-19 cases are in the most densely populated regions. However, not all densely populated areas had COVID-19 confirmed cases. The relative risk $(\mathrm{RR}>1)$ of secondary cases in a sector as compared with the overall risk in the country (SIR) showed that the contacts who tested positive for COVID-19 were also clustered in the southwestern part of Rwanda, City of Kigali and northeastern part of Rwanda (figure 5 (top right)).

Table 3 Top 20 sectors with higher number of contacts and their SIR

\begin{tabular}{|c|c|c|c|c|}
\hline Province & District & Sector & Contacts & SIR (95\% CI) \\
\hline Western & Rusizi & Kamembe & 2509 & 119.65 (90.85 to 154.68 ) \\
\hline Western & Rusizi & Gihundwe & 1527 & 64.59 (44.98 to 89.83$)$ \\
\hline Western & Rusizi & Mururu & 882 & 36.35 (21.16 to 58.20$)$ \\
\hline Western & Nyamasheke & Gihombo & 784 & 35.43 (20.63 to 56.73 ) \\
\hline Western & Rusizi & Butare & 244 & 18.34 (7.35 to 37.79$)$ \\
\hline Western & Rusizi & Nyakabuye & 368 & $14.47(6.23$ to 28.51$)$ \\
\hline Western & Rusizi & Giheke & 78 & 8.27 (1.66 to 24.16$)$ \\
\hline Kigali City & Kicukiro & Kigarama & 416 & 7.54 (3.02 to 15.54) \\
\hline Kigali City & Nyarugenge & Kimisagara & 278 & 6.62 (2.42 to 14.41$)$ \\
\hline Western & Rusizi & Nkombo & 160 & 6.13 (0.69 to 22.13 ) \\
\hline Kigali City & Gasabo & Jali & 140 & 5.54 (1.11 to 16.19$)$ \\
\hline Kigali City & Gasabo & Rusororo & 142 & 5.12 (1.38 to 13.11$)$ \\
\hline Kigali City & Nyarugenge & Kigali & 61 & 5.02 (1.01 to 14.67$)$ \\
\hline Southern & Nyanza & Busasamana & 149 & 3.5 (0.70 to 10.23$)$ \\
\hline Eastern & Nyagatare & Matimba & 14 & 3.49 (0.39 to 12.60$)$ \\
\hline Western & Rusizi & Nyakarenzo & 21 & 3.33 (0.04 to 18.53$)$ \\
\hline Kigali City & Nyarugenge & Rwezamenyo & 66 & 3.23 (0.04 to 17.97$)$ \\
\hline Kigali City & Gasabo & Remera & 242 & 3.18 (0.64 to 9.29$)$ \\
\hline Kigali City & Gasabo & Kimironko & 314 & 3.16 (0.85 to 8.09$)$ \\
\hline Kigali City & Kicukiro & Gikondo & 108 & 3.01 (0.04 to 16.75$)$ \\
\hline
\end{tabular}

SIR, standardised incidence rate. 
The area-specific probability figure 5 (top right and bottom) are based on a spatial model and uses information only from within the area; therefore, it might give uncertain estimates for small areas. We estimated relative risks that take into account the uncertainty and spatial association. Using this approach and stratifying by sex, we identified new clusters in northeast of Rwanda for both men and women. Among women, we identified another cluster in the southeast of Rwanda in addition to the one identified using SIR. The administrative map of sectors of Rwanda is provided as online supplemental figure S1.

\section{DISCUSSION}

Spread of COVID-19 in Rwanda largely occurred in Kigali (capital city). Age, male and sharing a home or office with an index case were factors driving the risk for secondary cases. In Rwanda, men accounted for $60 \%$ of all cases. At population level, markets/pubs/ shops were the places where the largest number of secondary cases occurred due to the large number of contacts being made at this place. Spread seemed to be driven by different age groups mixing, and younger individuals (20-29 and 30-39 years) seemed to drive the spread of the epidemic.

We found that in Rwanda, index cases and their contacts are clustered mainly in the City of Kigali, which is located in the central of Rwanda, in the southwest of country, Rusizi and Nyamasheke Districts. Among the first hundred COVID-19 cases in Rwanda, most were imported and identified as having recently travelled from Dubai, United Arab Emirates, which triggered contact tracing in City of Kigali. Regarding the Western province, the areas bordering Republic Democratic of Congo and Rwanda, Rusizi and Nyamashake had a SARS-CoV-2 community transmission due to legal and illegal cross-border movement during lockdown with inconsistency of using masks, keeping distance and washing hands regularly. Rusizi and Nyamasheke were the highest risk areas for COVID-19 in Rwanda. The contact tracing data showed that in the highest risk areas, the number of contacts per index case and per areas were higher as compared with other areas.

There was an increased transmission of infection among areas that were identified late as COVID-19 high-risk areas. The sectors of Rusizi and Nyamasheke Districts, such as Kamembe, Gihundwe and Mururu, recorded the highest number of identified contacts, as figure 5 (top left) showed in the map. Those sectors remained in lockdown for more than 3 months, while temporary localised lockdown duration in Rwanda was about 3 weeks maximum. We estimated relative risks and findings provided two new extra clusters in northeast for male and southeast for females. The spatial models can be useful in identifying clusters in low-risk COVID-19 areas. Spatial analysis is of great help in understanding the spread of infectious diseases. The spatial association was identified to be key during the early stages of the COVID-19 pandemic in mainland China. ${ }^{6}$

Five index cases with more than 200 contacts were identified. The first superspreader identified was a school teacher with 96 contacts, with among them 6 tested positive. Another one was taxi driver with 301 contacts of which five tested positive. The overall ratio of index case per secondary cases in this study is $8: 1$, while for the first superspreader is 1:6. Some other studies ${ }^{19}$ reported rapid increase of new cases linked to superspreaders and showed that with progress of diagnostic technology, potential superspreaders may be discovered in the future. ${ }^{20}$ We have observed that only $2.87 \%$ of the secondary cases had symptoms. In order to prevent the spread of SARS-CoV-2, asymptomatic spreaders need to be studied to detect them early. Though the overall AR is less than $2 \%$ among secondary cases, this varied by a number of factors. We found that AR was higher in men and people under 30 years old, household members living with index case and people attending social activities or workmates of index case.

The AR in Rwanda was smaller as compared with the AR in cities in China and USA (above 10\%). ${ }^{21}$ Unlike findings from other studies in Asia, ${ }^{22}$ the attack rate among the elderly was lower $(0.13 \%)$ in Rwanda for people aged above 60 years old. This might be partially explained by the fact that contacts aged 60 years and above could have less physical activities and movement as compared with people age 20-29 years old in Rwandan setting. In addition, Rwanda's population as a whole is quite young. Over $50 \%$ of Rwandans are under 20 years old, and the median age is 22.7 years old. ${ }^{23}$ We found that men experienced nearly twice the odds of infection compared with women. This finding differs from studies conducted elsewhere that observed female contacts that were more likely to be infected by SARS-CoV-2 than male contacts. ${ }^{9} 2224$

Despite the enforcement of prevention measures of COVID-19 in Rwanda, especially in public places, we observed that household members of index are two times at risk as compared with contacts that met index cases in public places such as market, shops or pubs (OR 1.92, 95\% CI 1.17 to 3.15). This could be explained by the fact that most of COVID-19 cases in Rwanda were asymptomatic, and several studies reported that both symptomatic and asymptomatic person are infectious. ${ }^{25}{ }^{26}$ Therefore, most infections might have been occurred unknowingly in families and relatives living in the same households.

Though Rwandans comply with several public health measures in place such as hand washing, universal face mask and physical distance, we have realised that more transmission occurred at markets. However, the individual risk appeared greater within the household (in spite of lockdowns). 


\section{CONCLUSION}

In summary, it is crucial to understand the transmission channels and situation of SARS-CoV-2 as a new virus to prevent and control its spreading in the community. The analysis of contact tracing data using spatial modelling allowed us to identify high-risk areas at subnational level in Rwanda. Estimating risk factors for infection with SARS-CoV-2 is vital in identifying the clusters in low spread of SARS-CoV-2 subnational level.

In a pandemic period, it is imperative to understand the interactions between the index case and contacts to identify superspreaders, risk factors and high-risk places. Understanding spread of the virus in a specific country leads to contextually appropriate interventions that slow the spread of SARS-CoV-2 and saves lives.

The findings recommend that self-isolation at home in Rwanda should be reviewed to limit secondary cases from the same households. The contact tracing strategy should include routine surveillance and testing of highrisk groups to limit community transmission. Spatial and spatiotemporal analysis should be introduced in routine monitoring of COVID-19 in Rwanda for policy making decision on real time.

\author{
Author affiliations \\ ${ }^{1}$ Center for Excellence in Data Science, University of Rwanda - Kigali Campus, \\ Kigali, Rwanda \\ ${ }^{2}$ Centre for Statistics, Hasselt Biostatistics and statistical Bioinformatics Center, \\ Diepenbeek, Limburg, Belgium \\ ${ }^{3}$ Applied Statistics, University of Rwanda College of Business and Economics - \\ Gikondo Campus, Kigali, Rwanda \\ ${ }^{4}$ Institute for HIV, Diseases Prevention and Control, Rwanda Biomedical Center, \\ Kigali, Rwanda \\ ${ }^{5}$ College of Medicine and Health Sciences, University of Rwanda, Kigali, Rwanda \\ ${ }^{6}$ Rwanda Biomedical Center, Rwanda Ministry of Health, Kigali, Rwanda \\ ${ }^{7}$ BioStat, Hasselt Biostatistics and statistical Bioinformatics Center, Diepenbeek, \\ Limburg, Belgium
}

Twitter Muhammed Semakula @semakulam

Acknowledgements The authors are grateful to Rwanda Biomedical Centre, COVID-19 Joint Task Force, frontliners and Data science, IT solutions team for technical support provided on data extraction. We are grateful to Rwanda Ministry of Health and Public Health Surveillance and Epidemic Preparedness and Response Division for authorising principal investigator to access data and sharing addition materials to understand contact tracing in Rwanda. CF acknowledges support from the European Union's innovation programme - project EpiPose (Grant agreement number 101003688).

Contributors MS and CF conceived the presented idea. MS developed the theory and performed the analysis. CF verified methods and validated the analysis. NF, $\mathrm{AU}, \mathrm{SN}, \mathrm{VD}, \mathrm{ER}, \mathrm{TN}$ and ER reviewed the manuscript. CF supervised the findings of this work. All authors discussed the results and contributed to the final manuscript.

Funding This study was funded by European Union's innovation programme project EpiPose (101003688).

Map disclaimer The depiction of boundaries on the map(s) in this article does not imply the expression of any opinion whatsoever on the part of BMJ (or any member of its group) concerning the legal status of any country, territory, jurisdiction or area or of its authorities. The map(s) are provided without any warranty of any kind, either express or implied.

Competing interests None declared.

Patient consent for publication Not required.

Provenance and peer review Not commissioned; externally peer reviewed.
Data availability statement Data are available in a public, open access repository. All data relevant to the study are included in the article or uploaded as supplementary information. Data sets used are available for public after manuscript is being accepted for publication.

Supplemental material This content has been supplied by the author(s). It has not been vetted by BMJ Publishing Group Limited (BMJ) and may not have been peer-reviewed. Any opinions or recommendations discussed are solely those of the author(s) and are not endorsed by BMJ. BMJ disclaims all liability and responsibility arising from any reliance placed on the content. Where the content includes any translated material, BMJ does not warrant the accuracy and reliability of the translations (including but not limited to local regulations, clinical guidelines, terminology, drug names and drug dosages), and is not responsible for any error and/or omissions arising from translation and adaptation or otherwise.

Open access This is an open access article distributed in accordance with the Creative Commons Attribution Non Commercial (CC BY-NC 4.0) license, which permits others to distribute, remix, adapt, build upon this work non-commercially, and license their derivative works on different terms, provided the original work is properly cited, appropriate credit is given, any changes made indicated, and the use is non-commercial. See: http://creativecommons.org/licenses/by-nc/4.0/.

ORCID iD

Eric Remera http://orcid.org/0000-0002-6084-8877

\section{REFERENCES}

1 Wadvalla B-A. How Africa has tackled covid-19. BMJ 2020;370:m2830.

2 Rosenthal PJ, Breman JG, Djimde AA, et al. COVID-19: shining the light on Africa. Am J Trop Med Hyg 2020;102:1145-8.

3 Republic of Rwanda, Ministry of Health. Coronavirus disease 2019, National preparedness and response plan. Available: https:// moh.gov.rw/fileadmin/user_upload/Publication/Coronavirus\% 20Disease\%202019\%2C\%20National\%20Protocol

4 RBC. Rwanda COVID-19 clinical managment guidelines 3rd edition, 2021. Available: https://www.rbc.gov.rw/fileadmin/user upload/guide/Guidelines/COVID 19\%20Clinical\%20Managment\% 20guidelines.pdf [Accessed 31 Mar 2021].

5 RezaPourghasemi H, Pouyan S, Heidari B, et al. Spatial modeling, risk mapping, change detection, and outbreak trend analysis of coronavirus (COVID-19) in Iran (days between February 19 and June 14, 2020) 2020;98:90-108.

6 Kang D, Choi H, Kim J-H, et al. Spatial epidemic dynamics of the COVID-19 outbreak in China. Int J Infect Dis 2020;94:96-102.

7 Shah K, Saxena D, Mavalankar D. Secondary attack rate of COVID-19 in household contacts: a systematic review QJM. Int J Infect Dis:hcaa232.

8 Karim N, Jing L, Lee JA, et al. Lessons learned from Rwanda: innovative strategies for prevention and containment of COVID-19. Ann Glob Health 2021;87:23.

9 Jing QLet al. Household secondary attack rate of COVID-19 and associated determinants in Guangzhou, China: a retrospective cohort study Lancet infect dis. 1103, 2020.

10 Semakula M, Niragire FI, Faes C. Bayesian spatio-temporal modeling of malaria risk in Rwanda. PLoS One 2020;15:e0238504-16.

11 Blangiordo M, Cameletti M. Spatial and Spatio temporal Bayesian models with R-INLA. UK: John Wiley \& Sons, 2015.

12 Lawson A, Lee D. Bayesian disease mapping for public health. In: Handbook of statistics, 2017: 443-81.

13 Lesaffre E, Lawson AB. Bayesian Biostatistics.Statistics in practice. UK: John Wiley \& Sons, 2012.

14 Besag J, York J, Molli A. Bayesian image restoration, with two applications in spatial statistics. Ann Inst Stat Math 1991;43:1-20. 1991 https://doi.org/10.1007/BF00116466

15 Besag J, Green PJ. Spatial statistics and Bayesian computation. Journal of the Royal Statistical Society: Series B 1993;55:25-37.

16 Carroll R, Lawson AB, Faes C, et al. Comparing INLA and OpenBUGS for hierarchical Poisson modeling in disease mapping. Spat Spatiotemporal Epidemiol 2015;14-15:45-54.

17 Giorgi E, Osman AA, Hassan AH, et al. Using non-exceedance probabilities of policy-relevant malaria prevalence thresholds to identify areas of low transmission in Somalia. Malar J 2018;17:88.

18 Agresti A. Categorical data analysis UK:John Wiley \& Sons. 3rd Edition, 2013. ISBN: 978-0-470-46363-5.

19 Sun P, Lu X, Xu C, et al. Understanding of COVID-19 based on current evidence. J Med Virol 2020;92:548-51. doi:10.1002/ jmv.25722 
20 Rothe Cet al. Transmission of 2019 nCov infection from an asymptomatic in Germany. NEngl JMed 2020;1468.

21 Luo Let al. Contact settings and risk for transmission in 3410 close contacts of patients with COVID-19 in Guangzhou, China, a prospective cohort study. Ann Intern Med 2020.

22 Liu T, Liang W, Zhong $\mathrm{H}$, et al. Risk factors associated with COVID-19 infection: a retrospective cohort study based on contacts tracing. Emerg Microbes Infect 2020;9:1546-53. doi:10.1080/222217 51.2020 .1787799

23 National institute of statistics. Population of Rwanda:Results from 4th census 2012.
24 pp.Bi Q, Wu Y, Mei S, et al. Epidemiology and transmission of COVID-19 in 391 cases and 1286 of their close contacts in Shenzhen, China: a retrospective cohort study. Lancet Infect Dis 2020;20:911-9.

25 Rothe C, Schunk M, Sothmann P, et al. Transmission of 2019-nCoV infection from an asymptomatic contact in Germany. N Engl J Med 2020;382:970-1.

26 Chan JF-W, Yuan S, Kok K-H, et al. A familial cluster of pneumonia associated with the 2019 novel coronavirus indicating personto-person transmission: a study of a family cluster. Lancet 2020;395:514-23. 